\title{
Probabilidade de Informação Privilegiada no Mercado de Ações, Liquidez Intra-Diária e Níveis de Governança Corporativa
}

\author{
Claudio Henrique Barbedo*, Eduardo Camilo da Silva ${ }^{\dagger}$, Ricardo \\ Pereira Câmara Leal ${ }^{\ddagger}$
}

Conteúdo:

Palavras-chave: Microestrutura; Probabilidade de Operação com Informação; Governança.

Códigos JEL: $\quad$ G14; C22.

Listagens de ações baseadas em práticas de Governança são utilizadas pelas bolsas de valores para sinalizar ao investidor uma maior proteção contra expropriações praticadas pelos grupos controladores. Neste trabalho, aproveitamos o experimento propício e único oferecido pela Bovespa e medimos a probabilidade de ocorrência de transações baseadas em informação (PIN) das ações listadas nos mercados Tradicional, Nível 1, Nível 2 e Novo Mercado. Os resultados confirmam a hipótese de que níveis mais altos de governança apresentam menor PIN. Adicionalmente, classes de listagens mais líquidas apresentaram PIN menor do que as menos líquidas devido, sobretudo, à maior presença de investidores não-informados. Non-traditional listing rules on stock exchanges based on corporate governance practices criteria may ensure investors a higher level of protection and constrain expropriation of minority shareholders. We investigate how distinct listing levels, according to the quality of corporate governance practices, a experiment provided by the Brazilian stock exchange (Bovespa), and liquidity levels are associated to the probability of informed trading (PIN). We find that PIN is lower in stricter corporate governance practices listing levels, after controlling for liquidity. Greater liquidity is associated to a lower PIN particularly due to the presence of non-informed investors.

* Doutor do Instituto COPPEAD de Administração da UFRJ. E-mail: cbarbedo@coppead.ufrj .br

${ }^{\dagger}$ Aluno de doutorado do Instituto COPPEAD de Administração da UFRJ. E-mail: ecamilo@mac.com

† Professor titular do Instituto COPPEAD de Administração da UFRJ. Rua 36, n. 355 - Ilha do Fundão. Caixa Postal 68514. Cep 21949-900 - Rio de Janeiro - RJ. E-mail: rleal@globo.com 


\section{INTRODUÇÃO}

A proteção ao acionista minoritário contra expropriação dos seus ganhos é fundamental para o funcionamento do mercado porque, em última análise, são eles que compõem o mercado. Entretanto, os casos de expropriação desses acionistas são inúmeros, conforme podemos ver em ? e muito mais freqüentes do que poderíamos supor, dada a relevância e a gravidade do tema. A razão para isso é o fato de que, na busca pela maximização da própria riqueza, o controlador enfrenta, em diversas decisões, conflitos gerados por situações em que a maximização da riqueza do acionista minoritário implicaria uma redução da sua própria.

Quando o uso ilegal da informação privilegiada permanece impune, como costuma ocorrer na maioria dos países da América Latina, os grupos controladores sentem-se confortáveis para expropriar os ganhos dos acionistas minoritários através do uso de sua informação privilegiada para negociar ações. Qualquer suspeita do investidor da ocorrência dessas atividades provoca a chamada seleção adversa, fazendo com que o investidor evite investir naquele mercado ou naquele ativo devido ao receio, plenamente justificado, de ser prejudicado na transação.

De fato, situações envolvendo conflito de interesses dentro de uma organização ocorrem em diversas esferas, tais como: acionistas versus executivos, controlador versus minoritário, empresa versus credores, empresa versus fornecedores etc. É do interesse das empresas sinalizar ao mercado que tais situações não ocorrem ou, pelo menos, que a empresa se empenha em evitar que elas ocorram em suas relações com seus acionistas. Às práticas e políticas adotadas voltadas para a prevenção dos conflitos de interesse, chamamos governança corporativa.

Para cada uma dessas relações com potencial para ocorrência de conflito de interesses, há uma lista de estratégias que são recomendadas por diversos autores e entidades. ${ }^{1}$ ?, ? e ?, entre outros, demonstraram que o mercado "precifica" a qualidade da governança corporativa de uma empresa. Daí o interesse das empresas em passar essa sinalização custosa do seu comportamento em relação às boas práticas de governança.

A criação de listagens de ativos selecionados pelas estratégias de governança adotadas pela empresas, como as listas N1, N2 e Novo Mercado da Bovespa, tem por objetivo passar ao investidor a confiança de que, ao comprar uma ação de um mercado com melhores práticas de governança, estará exposto a um menor risco de ser expropriado por agentes com informação privilegiada.

Grande parte das estratégias de governança corporativa - sejam elas ex ante, como regras e direito de voto na seleção de executivos, ou ex post, como a garantia de iguais condições da venda da empresa (tag along) ou direitos de remoção de conselheiros ou executivos - é voltada para o aumento e para a valorização da transparência ${ }^{2}$, que podemos entender como sendo o acesso dos investidores às informações relevantes para a precificação de suas ações. Essa palavra quase mágica aparece com muita freqüência em qualquer texto sobre governança, mas, apesar de tão utilizada, pouco foi feito para que pudesse ser efetivamente medida.

As únicas medidas voltadas para a medição da qualidade da governança corporativa de uma empresa são as referentes aos índices de governança corporativa, tais como os empregados por ? e ?, entre outros. Todos esses índices são montados com base na existência, ou não, de determinadas práticas, tais como a composição do conselho de administração ou direitos de voto dos minoritários. Ainda que estes índices tenham incontestável utilidade como indicador do nível de proteção que o investidor poderá obter ao investir nesta ou naquela empresa, eles, de fato, não oferecem uma medida objetiva da probabilidade de que o investidor venha a ser efetivamente expropriado.

Em outras palavras, os índices de Governança Corporativa medem o quanto das estratégias recomendadas é adotado, mas não medem a sua eficácia intrínseca nem como são aplicadas. Isto traz dois

\footnotetext{
${ }^{1}$ Ver ? e http: //www.ibgc.org.br.

${ }^{2}$ Segundo o Instituto Brasileiro de Governança Corporativa (IBGC), a boa Governança Corporativa assegura aos sócios: eqüidade, transparência, prestação de contas (accountability) e responsabilidade pelos resultados.
} 
problemas: o primeiro é não se saber se as estratégias realmente previnem a expropriação e o segundo é que, ainda que elas o façam, não se sabe se uma empresa em particular as aplica da maneira correta.

Considerando que a ocorrência de conflitos de interesse, tais como, a atuação de insider traders não é captada ex post pelos índices e que todas as estratégias acima atuam ex ante, percebemos que uma questão fundamental permanece: as práticas aplicadas segundo os regulamentos desses mercados efetivamente previnem a expropriação do acionista minoritário?

Criado por ?, a Probabilidade de Operação com Informação ou PIN (Probability of Informed Trading) procura medir (ex post) a probabilidade de que tenham ocorrido transações baseadas em informações privilegiadas.

? sugerem que a PIN serve como estimador da qualidade da governança corporativa conforme ela seja percebida pelo mercado através da análise de ações de empresas de sete países da América Latina, inclusive ADRs de empresas brasileiras.

Neste trabalho, aproveitamos este experimento natural único oferecido pela Bovespa e medimos a PIN de ações da BOVESPA listadas nos mercados Tradicional, N1, N2 e Novo Mercado, que se diferenciam pelo seu nível de práticas declaradas de Governança Corporativa, definido pelas regras para listagem em cada mercado, usando dados intra-diários.

Usamos a Probabilidade de Operação com Informação para comparar as diversas ações e os diversos mercados. Supostamente, os mercados de mais alto nível de governança deveriam apresentar menor probabilidade de ocorrências de transações baseadas em informação privilegiada. Os resultados, em níveis similares de liquidez, confirmam a hipótese. Entre as ações do mercado tradicional e as do nível 1, de nível similar de liquidez, as do nível 1 apresentam a menor PIN. Entre as ações do nível 2 e do Novo Mercado, estas últimas apresentam a menor PIN. Entretanto, as classes de listagem mais líquidas (tradicional e nível 1) apresentam PIN menor do que as classes de listagem menos líquidas (nível 2 e Novo Mercado), porque estas últimas apresentam menor ocorrência de investidores não informados com a menor liquidez. Se a liquidez do nível 2 e do Novo Mercado aumentar em cerca de $25 \%$, estes deveriam apresentar o mesmo valor de PIN que as classes de listagem mais líquidas.

O artigo está dividido da seguinte maneira: na Seção 2 apresentamos os modelos PIN utilizados no estudo. Na Seção 3, descrevemos a amostra e os resultados e a Seção 4 conclui o artigo.

\section{PROBABILIDADE DE OPERAÇÃO COM INFORMAÇÃO PRIVILEGIADA (PIN)}

? modelaram a probabilidade de informação privilegiada, baseadas nas negociações seqüenciais do mercado. Operações de compra e venda ocorrem por ações de operadores informados potenciais e desinformados. Informação, nesse caso, é definida como o sinal que pode ser interpretado como informação privilegiada sobre o verdadeiro valor do ativo. O modelo assume que tal sinal é o resultado de insider information.

Suponhamos que uma informação nova alcança o mercado sobre um ativo qualquer (um evento com informação) com probabilidade $\alpha$. Nesse caso, o sinal da informação pode ser baixo (notícia má) com probabilidade $\delta$ ou alto (notícia boa) com probabilidade $(1-\delta)$. Após a revelação dos sinais de informação, o ativo é negociado por operadores informados e desinformados que chegam ao mercado de acordo com processos independentes de Poisson. Os operadores desinformados não observam o sinal da informação e negociam independentemente da notícia. A cada dia, eles operam em ambos os lados do mercado, compra e venda, com uma taxa de chegada de $\epsilon^{3}$ O fato de os operadores informados comprarem ou venderem baseia-se na existência e no tipo de sinal da informação que observaram antes de a negociação começar. Eles negociam somente durante os dias em que o evento com informação ocorrer e somente em um lado do mercado. Se o sinal da informação for baixo, eles vendem; se o sinal da informação for alto, compram. A taxa de chegada de operadores informados é denotada por $\mu$.

${ }^{3} \mathrm{O}$ modelo assume igual proporção de compradores e vendedores desinformados. No trabalho original, ? testaram diferentes taxas e não encontraram diferença significativa nos modelos que justificassse uma alteração. 
As taxas de chegada $\epsilon$ e $\mu$ são definidas pelo número de negócios por dia de operadores desinformados e informados, respectivamente. A Figura ?? fornece uma apresentação gráfica desse processo de negociação.

Figura 1 - Modelo de ? para operações com informação privada

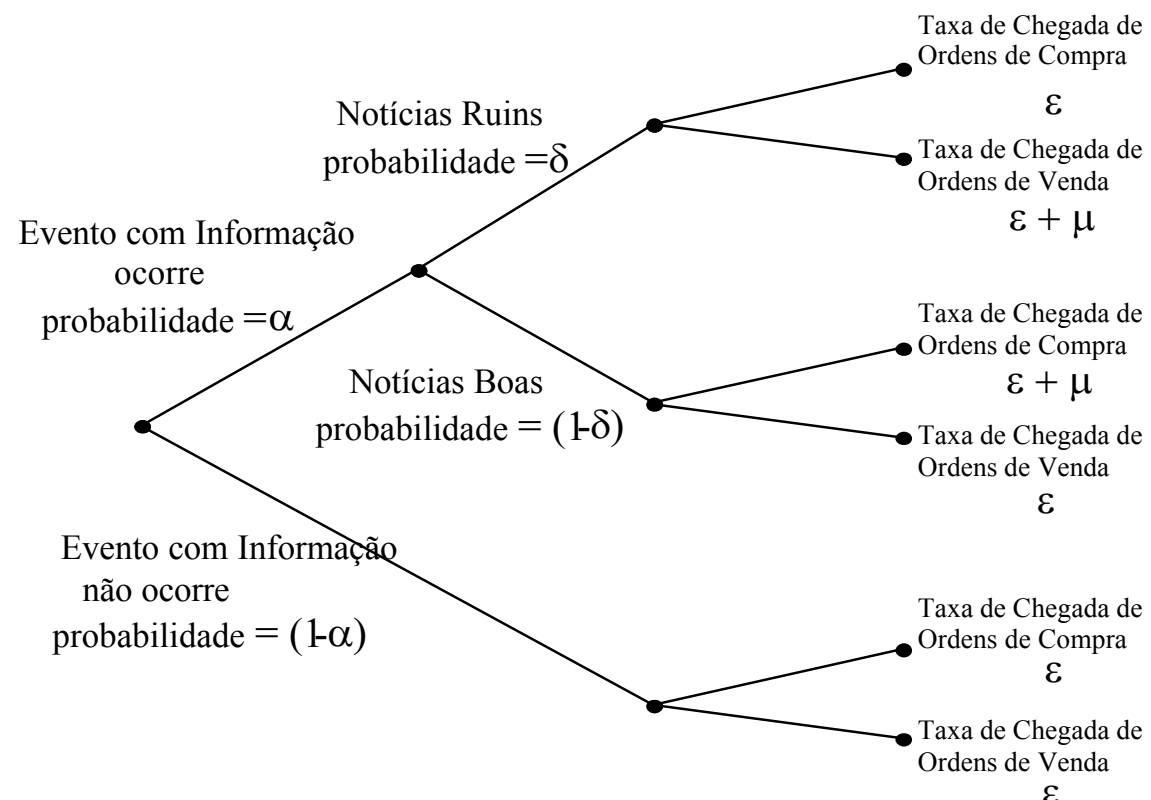

$\alpha$ é a probabilidade de ocorrer o evento com informação

$\delta$ é a probabilidade do evento com informação ser de notícia ruim

$\varepsilon$ é a taxa diária de operadores desinformados

$\mu$ é a taxa diária de operadores informados

A estrutura do modelo implica que mais ordens de compra são esperadas em dias de boas notícias, mais ordens de venda são aguardadas em dias de notícias ruins, enquanto poucas operações se esperam em dias sem eventos de informação, devido à diminuição de operadores informados no mercado. $\mathrm{O}$ desequilíbrio nos números das ordens de compra e de venda é justificado pela participação de operadores informados que participam somente em um lado do mercado, o que indica a Probabilidade de Operação com Informação. Os parâmetros de $\theta=\alpha, \delta, \epsilon, \mu$ não são diretamente observáveis, entretanto, como os processos de chegada das ordens de compra e de venda são observados, o modelo de ? constrói a função de verossimilhança de $\theta$, baseado nesses parâmetros.

O modelo supõe que as compras e as vendas seguem um dos três processos independentes de Poisson, dependendo se há notícias boas ou más, ou se não há notícias. Como não sabemos que processo está sendo operado em cada dia, a probabilidade resultante das chegadas das ordens é uma média ponderada da probabilidade de se observar um determinado número de ordens de compra ou venda em um dia de notícias boas, más ou em um dia sem notícias. Os pesos são probabilidades de cada tipo de situação ocorrida e são dados por $(1-\alpha)$ para um dia sem notícias, $\alpha \delta$ para dias de notícias ruins e $\alpha(1-\delta)$ para dias de notícias boas. A função de probabilidade para uma determinada ação é dada por: 


$$
L(B, S \mid \alpha, \epsilon, \delta, \mu)=\prod_{i=1}^{I}\left\{\begin{array}{l}
(1-\alpha)\left[e^{\left.-\epsilon T \frac{(\epsilon T)^{B_{i}}}{B_{i} !} e^{-\epsilon T} \frac{(\epsilon T)^{S_{i}}}{S_{i} !}\right]}\right. \\
+\alpha \delta\left[e^{\left.-\epsilon T \frac{(\epsilon T)^{B_{i}}}{B_{i} !} e^{-(\mu+\epsilon) T} \frac{[(\mu+\epsilon) T]^{S_{i}}}{S_{i} !}\right]}\right. \\
+\alpha(1-\delta)\left[e^{-(\mu+\epsilon) T} \frac{[(\mu+\epsilon) T]^{B_{i}}}{B_{i} !} e^{-\epsilon T} \frac{(\epsilon T)^{S_{i}}}{S_{i} !}\right]
\end{array}\right\}
$$

onde $B_{i}$ e $S_{i}$ representam o número de compras e vendas durante um período de tempo $T$, no dia $i$, e $\theta=(\alpha, \delta, \epsilon, \mu)$ é o vetor de parâmetros. Assumindo a independência entre os dias, obtemos a função de verossimilhança de $i=1, \ldots, I$ dias, calculando o produto das probabilidades diárias. A maximização da função obtém estimativas da taxa de operadores informados e desinformados ( $\mu$ ou $\epsilon$ ), bem como o tipo de evento para a ação $(\alpha$ ou $\delta)$. Um nível de ordens de compra e venda balanceado é interpretado como operações desinformadas e é usado para identificar $\epsilon$, enquanto o desequilíbrio é usado para identificar $\mu$. Para um determinado dia, a estimativa da probabilidade dos parâmetros de evento com informação $\alpha$ e $\delta$ é 0 ou 1, refletindo a ocorrência ou não do evento. Esses parâmetros são usados, então, para determinar a probabilidade de uma operação conter informação, a Probabilidade de Operação com Informação, dada por ${ }^{4}$

$$
P I=\frac{\alpha \mu}{\alpha \mu+2 \epsilon}
$$

A Equação acima indica que esta probabilidade aumenta com os eventos de informação (capturado por $\alpha$ ), aumenta com o número de traders informados (capturados por $\mu$ ) e diminui com o número de traders desinformados (capturados por $\epsilon$ ). No entanto, evidências empíricas sugerem a necessidade de se incorporarem correlações de seção reta dos fluxos das ordens, de maneira a tornar o modelo mais aderente à realidade. Por exemplo, o maior número de compras pode estar ligado a uma tendência de alta do mercado (que pode ser captada pelo índice de ações), assim como o maior número de vendas pela tendência contrária. Dado que o mercado de ações se comporta nitidamente por tendências de alta ou baixa decorrente do momento econômico ou de riscos estruturais, adotamos, neste trabalho, o ajuste de ?, no qual há uma classificação de que o dia da operação pode ser um dia de tendência de baixa (com probabilidade $\gamma$ ) ou de alta $(1-\gamma)$. No caso de alta, o modelo assume que as taxas de chegada das ordens de compra ou venda são acrescidas por uma constante ö. Para estimar os parâmetros, utilizamos as quantidades de compra e venda da ação para encontrar os parâmetros do primeiro modelo. A seguir, agregamos as quantidades de compra $\left(B_{i}\right)$ e venda $\left(S_{i}\right)$ do índice de ações para obter uma estimativa das novas variáveis. A nova função de probabilidade para uma determinada ação passa a ser dada por

$$
\left.\begin{array}{l}
L\left(B_{I}, S_{I} \mid \phi, \gamma\right)= \\
\left.\prod_{i=1}^{I}\left\{\begin{array}{c}
(1-\gamma)\left\{\begin{array}{l}
(1-\alpha)\left[e^{(-2(\theta+\epsilon)) T} \frac{[T(\epsilon+\phi)]^{B_{i}}}{B_{i} !} \frac{[T(\epsilon+\phi)]^{S_{i}}}{S_{i} !}\right]+ \\
\alpha \delta\left[e^{(-2(\theta+\epsilon)+\mu) T} \frac{(T(\epsilon+\phi))^{B_{i}}}{B_{i} !} \frac{[(\mu+\epsilon+\phi) T]^{S_{i}}}{S_{i} !}\right]+ \\
\alpha(1-\delta)\left[e^{\left.(-2(\theta+\epsilon)+\mu) T \frac{[(\mu+\epsilon+\phi) T]^{B_{i}}}{B_{i} !} \frac{[(\epsilon+\phi) T]^{S_{i}}}{S_{i} !}\right]}\right.
\end{array}\right\} \\
+ \\
\gamma\left\{(1-\alpha)\left[e^{-\epsilon T} \frac{(\epsilon T)^{B_{i}}}{B_{i} !} e^{-\epsilon T} \frac{(\epsilon T)^{S_{i}}}{S_{i} !}\right]+\alpha \delta\left[e^{-\epsilon T} \frac{(\epsilon T)^{B_{i}}}{B_{i} !} e^{-(\mu+\epsilon) T} \frac{[(\mu+\epsilon) T]^{S_{i}}}{S_{i} !}\right.\right.
\end{array}\right]\right\}
\end{array}\right\}
$$

Por esse novo modelo, a Probabilidade de Operação com Informação diminui com $\phi$ e passa a ser dada por

$$
P I=\frac{\alpha \mu}{\alpha \mu+2(\epsilon+(1-\gamma) \phi)}
$$

\footnotetext{
${ }^{4} \mathrm{~A}$ probabilidade é válida somente para o período em que há negociação, ou seja, o período em que o market maker ou outro agente eventual precifica a probabilidade de um evento com informação.
} 


\section{AMOSTRA E RESULTADOS}

A quantidade de ordens de compra e venda diária foi extraída a partir de dados intra-diários que continham os horários de negociação de traders e corretoras da Bovespa. ${ }^{5} 0$ tratamento de dados consistiu em, a partir dos horários intra-diários, identificar a origem de cada operação para montar um banco de dados de quantidades de ordens de compra e venda de cada ativo estudado. ${ }^{6}$ Foram selecionadas 48 ações com práticas diferenciadas de governança corporativa nos níveis 1 e 2, Novo Mercado e tradicional. O objetivo era formar 4 carteiras, uma de cada mercado, cada uma com 12 ações, sendo as 6 ações mais líquidas do ano de 2006 e as 6 menos líquidas. A amostra de dados compreende o período de 2 de janeiro de 2001 a 30 de junho de 2006. O número limitado de ações se dá devido ao elevado número de observações de alta freqüência das mesmas. A Tabela ?? apresenta as empresas estudadas e a medida de liquidez utilizada. ${ }^{7}$

Os parâmetros do modelo PIN são obtidos pela maximização das funções de probabilidade (1) e (2). A estimativa dos parâmetros $\alpha$ e $\delta$ é restrita no intervalo $[0,1]$, enquanto $\epsilon$ e $\mu$ são restritos em $[0,8)$. Foi utilizado um programa desenvolvido no S-Plus.

As empresas mais líquidas de cada categoria de listagem são significativamente mais líquidas, de acordo com o índice de liquidez da Bovespa, do que as menos líquidas. As empresas de alta liquidez da listagem tradicional e do N1 são significativamente diferentes em termos de liquidez das empresas do NM e do N2. Entretanto, as empresas de alta liquidez do grupo tradicional e N1 não são significativamente diferentes entre si, bem como as dos grupos N2 e NM. Para as empresas de baixa liquidez, a diferença significativa se dá entre as empresas da listagem tradicional e do N1 e do N2. De forma geral, podemos dizer que os grupos tradicional e N1 são bem próximos entre si e distantes do grupo N2 e NM em termos de liquidez, que, por sua vez, também são próximos entre si. Essas estatísticas não são mostradas neste artigo, mas podem ser requisitadas aos autores.

\subsection{Resultados da Estimação}

A estimativa da PIN e dos parâmetros estão apresentados na Tabela 2. Devido à não-normalidade da distribuição dos parâmetros, a apresentação dos resultados será descrita com base na mediana. Além disso, utilizamos o teste não-paramétrico de Kruskal-Wallis para verificar a hipótese nula de que os parâmetros das ações do nível 1, nível 2 e Novo Mercado têm a mesma distribuição, simultaneamente.

As ações do mercado tradicional apresentam a menor probabilidade de operação com informação. Dos mercados com práticas diferenciadas de governança, as ações do nível 1 apresentam a menor probabilidade-média de operação com informação, 0,0831, seguidas pelas do Novo Mercado, 0,1526 e, por último, pelas do nível 2. O teste não paramétrico de Kruskal-Wallis rejeita a hipótese nula de que os parâmetros tenham a mesma distribuição.

A probabilidade de ocorrer um evento com informação $\alpha$ é menor para as ações do mercado tradicional, entretanto o teste de Kruskal-Wallis não rejeita a hipótese nula de que os parâmetros têm a mesma distribuição que os do nível 1. As probabilidades médias de evento com informação para o Novo Mercado, 0,4932, e nível 2, 0,5214, são consideravelmente altas.

Em relação à probabilidade de o evento com informação ser de notícia ruim $(\delta)$ e à taxa de chegada de investidores informados $(\mu)$, o teste de Kruskal-Wallis não rejeita a hipótese nula de que os parâmetros dos 4 mercados tenham a mesma distribuição. Em relação à taxa de chegada de investidores

\footnotetext{
${ }^{5} \mathrm{Em}$ relação à base de dados, gostaríamos de agradecer aos Srs. Ricardo Nogueira, Rogério Marques e à sua equipe técnica.

${ }^{6}$ Para cada mês, tínhamos cerca de 1.000 .000 de registros de transações de compra e venda.

${ }^{7}$ Utilizou-se um índice de liquidez em bolsa fornecido pela Economática e calculado pela Bovespa. A medida considera o número de dias no período em que se teve pelo menos um negócio com a ação da firma, o número de negócios e o volume em dinheiro com os papéis da firma no período e o número de negócios e o volume em dinheiro com todos os papéis da bolsa. A fórmula empregada pode ser vista no website da Bovespa ou no sistema Economática.
} 
Tabela 1 - Empresas utilizadas para a formação de carteiras dos mercados tradicional (Trad), Novo mercado (NM), Nível 1 e Nível 2 e Índice de liquidez no ano de 2006

\begin{tabular}{|c|c|c|c|c|c|c|c|}
\hline Empresa & Classe & Liquidez & Mercado & Empresa & Classe & Liquidez & Mercado \\
\hline Petrobras & PN & 9,6071 & Trad & Vale Rio Doce & PNA & \begin{tabular}{|l|}
6,7681 \\
\end{tabular} & Nível 1 \\
\hline Telemar Par & PN & 4,6248 & Trad & Bradesco & PN & \begin{tabular}{|l|}
3,6033 \\
\end{tabular} & Nível 1 \\
\hline Usiminas & PNA & 3,8601 & Trad & Itaú Hold. & PN & 2,6651 & Nível 1 \\
\hline Sid Nacional & ON & 2,6765 & Trad & Gerdau & PN & 2,4872 & Nível 1 \\
\hline Caemi & PN & 2,4517 & Trad & Braskem & PNA & 2,1819 & Nível 1 \\
\hline Eletrobras & PNB & 1,8425 & Trad & Cemig & PN & 1,7788 & Nível 1 \\
\hline Telefônica Hold. & PN & 0,0078 & Trad & Unibanco & ON & 0,019 & Nível 1 \\
\hline Petroq. União & $\mathrm{PN}$ & 0,0076 & Trad & Itausa & ON & 0,016 & Nível 1 \\
\hline Tele Norte Cel. & ON & 0,0073 & Trad & Unibanco & PN & 0,0133 & Nível 1 \\
\hline F Cata & PNA & 0,0072 & Trad & els & $\mathrm{PN}$ & 0,0081 & Nível 1 \\
\hline M\&G Poliest & ON & 0,0071 & Trad & Aracruz & ON & 0,0075 & Nível 1 \\
\hline Tele Sudeste Cel. & ON & 0,0067 & Trad & Braskem & ON & 0,0071 & Nível 1 \\
\hline CCR Rodovias & ON & 0,7965 & NM & Net & $\mathrm{PN}$ & 1,868 & \begin{tabular}{|l|} 
Nível 2 \\
\end{tabular} \\
\hline Sabesp & $\mathrm{ON}$ & 0,6432 & NM & Celesc & PNB & 0,601 & Nível 2 \\
\hline Natura & $\mathrm{ON}$ & 0,5891 & $\mathrm{NM}$ & TAM & $\mathrm{PN}$ & 0,5502 & Nível 2 \\
\hline Light & $\mathrm{ON}$ & 0,5242 & $\mathrm{NM}$ & ALL A. Latina & UNT & 0,5145 & Nível 2 \\
\hline Perdigão & $\mathrm{PN}$ & 0,4963 & $\mathrm{NM}$ & Gol & $\mathrm{PN}$ & 0,4661 & \begin{tabular}{|l|} 
Nível 2 \\
\end{tabular} \\
\hline Cosan & $\mathrm{ON}$ & 0,4322 & $\mathrm{NM}$ & Eletropaulo & $\mathrm{PN}$ & 0,3936 & \begin{tabular}{|l|} 
Nível 2 \\
\end{tabular} \\
\hline Porto Seguro & $\mathrm{ON}$ & 0,1372 & $\mathrm{NM}$ & UOL & $\mathrm{PN}$ & 0,1545 & \begin{tabular}{|l|} 
Nível 2 \\
\end{tabular} \\
\hline Gafisa & $\mathrm{ON}$ & 0,0822 & NM & Marcopolo & $\mathrm{PN}$ & 0,1049 & \begin{tabular}{|l|} 
Nível 2 \\
\end{tabular} \\
\hline Copasa MG & $\mathrm{ON}$ & 0,0504 & NM & Eternit & ON & 0,0813 & \begin{tabular}{|l|} 
Nível 2 \\
\end{tabular} \\
\hline Company & ON & 0,0238 & NM & Saraiva Livr & $\mathrm{PN}$ & 0,0507 & Nível 2 \\
\hline Totvs & ON & 0,0217 & NM & Vivax & UNT & 0,0426 & Nível 2 \\
\hline American Banknote & ON & 0,0095 & NM & Equatorial & UNT & 0,0116 & Nível 2 \\
\hline
\end{tabular}

desinformados $(\epsilon)$, encontramos diferença significativa entre os mercados. Enquanto a mediana para os mercados tradicional e nível 1 é de 54,45 e 52,12, a mediana do Novo Mercado e do nível 2 é de 48,59 e 43,61. Isso significa que o problema da maior probabilidade de operação com informação nesses mercados não está somente ligado à presença de operadores com informação privilegiada, mas também à ausência de operadores ou investidores desinformados. Os testes realizados sobre a igualdade de liquidez já haviam indicado que a listagem tradicional e o Nível 1 são diferentes tanto do Nível 2 quanto do Novo Mercado, apresentando maior liquidez, e, portanto, mais operadores sem informação. 0 teste de Kruskal-Wallis rejeita a hipótese nula de que os parâmetros tenham a mesma distribuição. Em termos de consistência da PIN, o desvio-padrão é maior para as empresas do Novo Mercado e menor para o Nível 1.

Simulações com os números do período de 2001 a 2006 mostram que um aumento na taxa de chegada de investidores desinformados de cerca de $25 \%$ é suficiente para que o Novo Mercado e o nível 2 apresentem probabilidades de operação com informação similares às do mercado tradicional e nível 1. Uma maior probabilidade de operação com informação decorrente da escassez de investidores desinformados é consistente com os trabalhos de ? e ?.

A Figura ??, a seguir, mostra o comportamento das probabilidades de operação com informação ao longo do tempo. As variáveis foram estimadas em períodos de 60 dias. 
Tabela 2 - Estatística da probabilidade de operação com informação em carteiras de ações do Nível 1 (N1), Nível 2 (N2), Novo Mercado (NM) e Tradicional (Trad). P-valores reportam resultados do teste de Kruskal-Wallis

\begin{tabular}{|c|c|c|c|c|c|}
\hline \multirow{9}{*}{$\begin{array}{c}\alpha \\
\text { (prob. de } \\
\text { evento } \\
\text { com informação) }\end{array}$} & Estatística & Trad & N1 & N2 & NM \\
\hline & Média & 0,304 & 0,332 & 0,521 & 0,493 \\
\hline & Mediana & 0,309 & 0,342 & 0,527 & 0,469 \\
\hline & Máximo & 0,581 & 0,455 & 0,687 & 10,000 \\
\hline & Mínimo & 0,067 & 0,112 & 0,314 & 0,218 \\
\hline & Desvio-Padrão & 0,125 & 0,095 & 0,105 & 0,197 \\
\hline & $1 \mathrm{Q}$ & 0,214 & 0,287 & 0,452 & 0,380 \\
\hline & $3 Q$ & 0,374 & 0,404 & 0,589 & 0,519 \\
\hline & & & P-Valor & 0,000 & \\
\hline \multirow{8}{*}{$\begin{array}{c}\delta \text { (Prob. de notícias } \\
\quad \text { ruins, dado a) }\end{array}$} & Média & 0,392 & 0,301 & 0,367 & 0,423 \\
\hline & Mediana & 0,374 & 0,257 & 0,356 & 0,411 \\
\hline & Máximo & 0,766 & 0,735 & 0,595 & 0,664 \\
\hline & Mínimo & 0,092 & 0,021 & 0,199 & 0,114 \\
\hline & Desvio-Padrão & 0,184 & 0,209 & 0,115 & 0,153 \\
\hline & $1 \mathrm{Q}$ & 0,279 & 0,139 & 0,287 & 0,339 \\
\hline & $3 Q$ & 0,547 & 0,463 & 0,459 & 0,550 \\
\hline & & & P-Valor & 0,156 & \\
\hline \multirow{8}{*}{$\begin{array}{c}\mu \text { (Taxa de } \\
\text { operadores } \\
\text { informados) }\end{array}$} & Média & 34,903 & 32,754 & 35,325 & 35,193 \\
\hline & Mediana & 33,604 & 33,662 & 35,278 & 33,353 \\
\hline & Máximo & 57,779 & 47,924 & 47,800 & 49,633 \\
\hline & Mínimo & 21,810 & 20,044 & 10,000 & 19,312 \\
\hline & Desvio-Padrão & 9,942 & 6,977 & 7,364 & 8,529 \\
\hline & $1 \mathrm{Q}$ & 26,823 & 28,641 & 33,385 & 30,159 \\
\hline & $3 Q$ & 42,144 & 35,470 & 37,395 & 42,649 \\
\hline & & & P-Valor & 0,292 & \\
\hline \multirow{8}{*}{$\begin{array}{c}\epsilon \text { (Taxa de } \\
\text { operadores } \\
\text { não informados) }\end{array}$} & Média & 53,990 & 52,998 & 43,240 & 46,866 \\
\hline & Mediana & 54,452 & 52,126 & 43,612 & 48,593 \\
\hline & Máximo & 60,970 & 62,779 & 54,961 & 55,642 \\
\hline & Mínimo & 46,533 & 47,865 & 10,000 & 29,188 \\
\hline & Desvio-Padrão & 3,613 & 4,064 & 8,594 & 6,825 \\
\hline & $1 \mathrm{Q}$ & 50,967 & 50,663 & 41,272 & 45,327 \\
\hline & $3 Q$ & 56,351 & 53,937 & 46,878 & 51,671 \\
\hline & & & P-Valor & 0,000 & \\
\hline \multirow{8}{*}{ PIN } & Média & 0,083 & 0,089 & 0,176 & 0,153 \\
\hline & Mediana & 0,083 & 0,090 & 0,183 & 0,136 \\
\hline & Máximo & 0,163 & 0,120 & 0,242 & 0,289 \\
\hline & Mínimo & 0,034 & 0,044 & 0,097 & 0,080 \\
\hline & Desvio-Padrão & 0,030 & 0,020 & 0,036 & 0,056 \\
\hline & $1 \mathrm{Q}$ & 0,065 & 0,076 & 0,162 & 0,118 \\
\hline & $3 Q$ & 0,103 & 0,103 & 0,199 & 0,194 \\
\hline & & & P-Valor & 0,000 & \\
\hline
\end{tabular}


Figura 2 - Probabilidade de operação com informação em carteiras de ações dos Níveis 1 e 2 (N1 e N2), Novo Mercado (NM) e Tradicional (Trad)

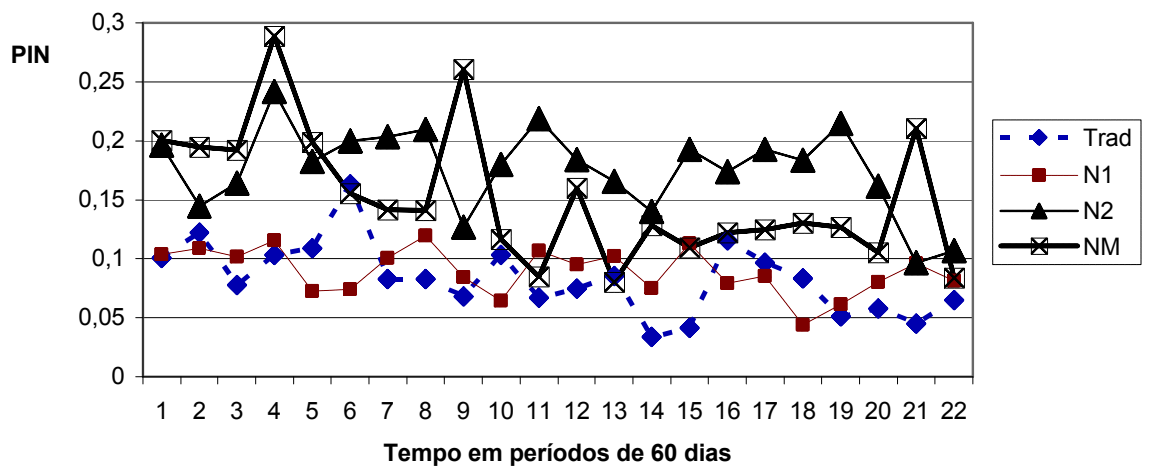

Pela Figura ??, constatamos que as carteiras compostas por ações dos mercados tradicional e nível 1 apresentam as menores probabilidades de operação com informação durante todo o período de estudo.

Para levar em consideração as possíveis tendências de alta ou baixa do mercado no comportamento das quantidades de ordens de compra e venda, estimamos novas probabilidades de operação com informação filtrando os dados, segundo a função de probabilidade da equação 2, por uma carteira formada pelas ações do Ibovespa. O objetivo é verificar se, neste caso, o comportamento das probabilidades se mantém, ou seja, as menores probabilidades de informação continuam nos mercados tradicional e nível 1.

A Figura ?? apresenta os resultados classificados individualmente para cada ação estudada. Valores maiores no eixo das ordenadas significam que a ação carrega uma maior probabilidade de as operações de compra ou venda realizadas nos últimos 5 anos terem sido estimuladas por uma informação diferenciada do operador.

Figura 3 - Probabilidade de Operação com Informação das Ações do Nível 1 (N1), Nível 2 (N2), Novo Mercado (NM) e Tradicional (Trad), Filtradas pela Carteira Composta por Ações do Índice Bovespa

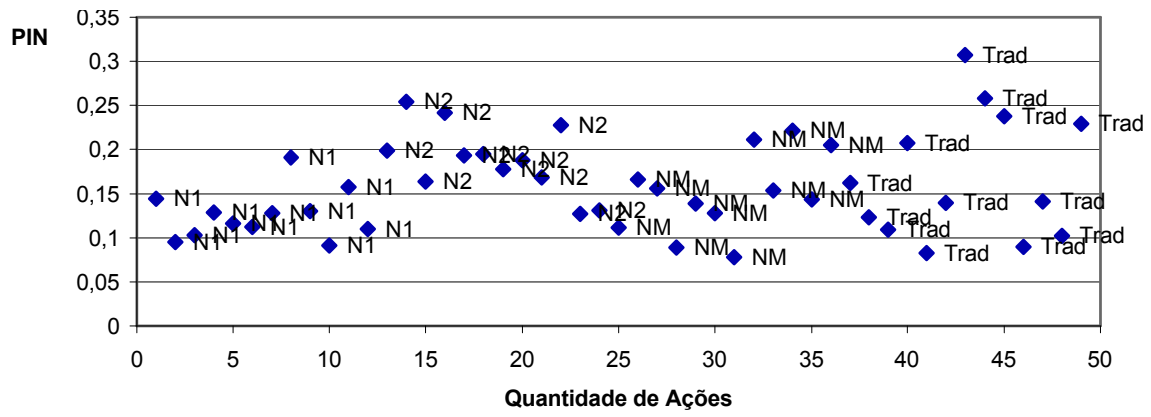

Pela Figura ??, verificamos que o melhor desempenho, isto é, as menores probabilidades de operação com informação, são verificadas no nível 1. A Tabela ?? apresenta a nova estatística de cada mercado, inicialmente com uma carteira composta pelas seis ações mais líquidas, pelas seis ações menos líquidas e pelo total de ações. 
Tabela 3 - Estatística das Probabilidades de Operação com Informação das Ações dos Níveis 1 e 2, Novo Mercado e Tradicional, Filtradas pela Carteira Composta por Ações do Índice Bovespa. A primeira parte da tabela apresenta as estatísticas das seis ações mais líquidas de cada mercado, a segunda, as seis ações menos líquidas e a terceira apresenta as estatísticas para o total das ações estudadas

\begin{tabular}{|lcccc|}
\hline \multicolumn{5}{|c|}{ Carteira Composta pelas Seis Ações mais Líquidas de cada Mercado } \\
\hline Estatística & $\mathrm{N} 1$ & $\mathrm{~N} 2$ & $\mathrm{NM}$ & Trad \\
\hline Média & 0,1148 & 0,1869 & 0,1402 & 0,1273 \\
Mediana & 0,114 & 0,1969 & 0,1483 & 0,1163 \\
Desvio-Padrão & 0,0124 & 0,0597 & 0,0498 & 0,0352 \\
\hline Carteira Composta pelas & Seis Ações & menos Líquidas de cada Mercado \\
\hline Estatística & $\mathrm{N} 1$ & $\mathrm{~N} 2$ & $\mathrm{NM}$ & Trad \\
\hline Média & 0,1361 & 0,1748 & 0,1601 & 0,2152 \\
Mediana & 0,1371 & 0,1781 & 0,1474 & 0,2228 \\
Desvio-Padrão & 0,0368 & 0,0429 & 0,0441 & 0,0663 \\
\hline \multicolumn{5}{|c|}{ Carteira Composta pelo Total das Ações } \\
\hline Estatística & $\mathrm{N} 1$ & $\mathrm{~N} 2$ & $\mathrm{NM}$ & Trad \\
\hline Média & 0.1236 & 0.1929 & 0.1610 & 0.1626 \\
Mediana & 0.1084 & 0.1842 & 0.1494 & 0.1334 \\
Máximo & 0.5564 & 0.5987 & 0.2987 & 0.6718 \\
Mínimo & 0.0000 & 0.0412 & 0.0780 & 0.0304 \\
Desvio-Padrão & 0.0685 & 0.0742 & 0.0488 & 0.0985 \\
1Q & 0.0875 & 0.1418 & 0.1280 & 0.0907 \\
3Q & 0.1374 & 0.2285 & 0.1991 & 0.1996 \\
\hline
\end{tabular}

As ações do Novo Mercado são as mais homogêneas, por apresentarem valores de máximo e mínimo próximos e o menor desvio-padrão, o que significa probabilidades mais estáveis ao longo do tempo. A divisão entre ações mais e menos líquidas também demonstram essa homogeneidade no Novo Mercado. De um modo geral, as ações do nível 1 são as que apresentam menor probabilidade de operação com informação, bem como as do mercado tradicional. Entretanto, a listagem tradicional, como seria de se esperar, apresenta a maior diferença entre as ações mais e menos líquidas, o maior desvio-padrão e a maior diferença no desvio-padrão entre as mais e menos líquidas. Assim como na Tabela 2, as ações do nível 2 apresentam a maior probabilidade de operação com informação na amostra total, mas, quando segmentamos por liquidez, a maior probabilidade fica com as ações menos líquidas da listagem tradicional.

O que se percebe, a partir dos resultados relatados, é que a liquidez importa muito na ocorrência de operações com informação. Quanto maior a liquidez, menor a probabilidade de operação com informação e maior a participação de operadores não-informados, como seria de se esperar. Os níveis de governança corporativa influenciam no resultado, uma vez considerada a liquidez. Assim, quando consideramos que a liquidez da listagem tradicional e do nível 1 é similar, elas se distinguem, em termos de probabilidade de operação com informação, por suas práticas de governança corporativa, uma vez que tal probabilidade é mais alta na listagem tradicional, na qual, supostamente, as práticas não são tão boas quanto no nível 1 , onde tal probabilidade é mais baixa. O mesmo se passa com o grupo composto pelo nível 2 e pelo Novo Mercado. Essas duas categorias de listagem são similares em termos de liquidez e apresentam probabilidade de operação com informação mais alta do que o grupo mais 
líquido formado pelo nível 1 e pela listagem tradicional. Entretanto, tal probabilidade é menor no Novo Mercado do que no nível 2. Sendo assim, podemos concluir que, para grupos de ações de liquidez similar, quanto melhores as práticas de governança corporativa, menor será a probabilidade de operação com informação. Os p-valores apresentados na Tabela ?? ratificam as similaridades do nível 1 com o tradicional e do nível 2 com o Novo Mercado em relação a PIN. Os p-valores acima de 5\%, do teste t, indicam que as listagens não são significativamente diferentes.

Tabela 4 - P-valores do Teste $t$, cuja Hipótese Nula é a de que as Médias das PIN’s das Listagens Indicadas são Diferentes

\begin{tabular}{|cc|}
\hline \multicolumn{2}{|c|}{ Carteira Composta pelas Seis Ações mais Líquidas de cada Mercado } \\
\hline Mercados & P-Valor \\
\hline N1 x N2 & 0,02346 \\
N1 x NM & 0,01616 \\
N1 x Trad & 0,06376 \\
N2 x NM & 0,55297 \\
\hline Carteira Composta pelas Seis Ações menos Líquidas de cada Mercado \\
\hline Mercados & P-Valor \\
\hline N1 x N2 & 0,02875 \\
N2 x Trad & 0,0232 \\
N1 x Trad & 0,07774 \\
N2 x NM & 0,51318 \\
\hline
\end{tabular}

\section{CONCLUSÃO}

Este artigo se propôs a estimar a probabilidade de operação com informação privilegiada no mercado de ações da BOVESPA, dividido de acordo com seu nível de Governança Corporativa. Foram investigados o mercado tradicional, o nível 1, o nível 2 e o Novo Mercado. Era esperado que os mercados de mais alto nível de governança apresentassem menor probabilidade de ocorrências de transações baseadas em informação privilegiada.

O modelo de estimativa de probabilidade de operação com informação privilegiada foi desenvolvido por ? para estimar, ex post, a probabilidade de terem ocorrido transações baseadas em informações privilegiadas. $\mathrm{O}$ trabalho incorpora uma alteração no modelo, um ajuste de tendência global de compra ou venda para filtrar as operações de acordo com o momento de alta ou baixa do mercado. O "filtro" escolhido é uma carteira formada pelas ações do Ibovespa.

Os resultados indicam que ações do mercado tradicional apresentam a menor probabilidade de operação com informação, seguida das ações do nível 1 e pelas do Novo Mercado e nível 2. Os resultados são fortemente influenciados pelo nível de liquidez. Os resultados piores para o Novo Mercado e o nível 2 se devem a dois fatores:

1. A maior probabilidade de ocorrer um evento com informação e

2. A baixa taxa de chegada de investidores desinformados.

Esse problema está associado à liquidez. Dentro de níveis de liquidez similares, os níveis de listagem com melhores práticas de governança corporativa apresentam menores probabilidades de operação com informação. 
No caso da taxa de chegada de investidores informados, notamos que um aumento de $25 \%$ dessa taxa no N2 e no Novo Mercado seria suficiente para, estatisticamente, igualar a sua probabilidade de operações com informação à probabilidade do mercado tradicional. Com mais liquidez era de se esperar, a ocorrência de mais investidores não-informados no mercado tradicional. A reversão dessa tendência precisa ser buscada para a redução da probabilidade de operações com informação por meio do aumento da liquidez das empresas nos níveis de listagem com práticas diferenciadas de governança corporativa.

Apesar de a PIN ser bem conhecido e desenvolvido na literatura financeira, este é o primeiro trabalho que se propõe a testar e a apresentar essa medida no mercado brasileiro. Por outro lado, a Bovespa oferece um experimento natural, único, com seus níveis diferenciados de governança corporativa. Não conhecemos outros trabalhos no âmbito internacional que tenham aplicado essa técnica a experimentos naturais similares.

A PIN também pode ser usado para a estimação da eficácia de ações de governança tomadas individualmente e em conjunto; esse estudo pode orientar a alocação dos investimentos em governança de modo a maximizar seus resultados. 